\title{
C-reactive protein as a prognostic marker in coronavirus disease-2019
}

\author{
Tarana Sarwat ${ }^{1}$, Devendra Kumar ${ }^{2}$, Neeraj Pal Singh ${ }^{3}$, Dalip Kakru ${ }^{4}$ \\ From ${ }^{1}$ Assistant Professor, Department of Microbiology, ${ }^{2}$ Associate Professor, Department of Respiratory Medicine, ${ }^{3}$ Assistant Professor, Department \\ of Community Medicine, ${ }^{4}$ Professor, Department of Microbiology, SMS and R, Sharda University, Greater Noida, India
}

Correspondence to: Tarana Sarwat, Department of Microbiology, SMS and R, Sharda University, Greater Noida, India. E-mail: taranasf@gmail.com

Received - 05 Aug 2021

Initial Review - 22 Aug 2021

Accepted - 30 Aug 2021

\section{ABSTRACT}

Background: Coronavirus disease-2019 (COVID-19) is a recently emerged viral disease, for which there's currently no definitive treatment. It is, therefore, necessary to determine biomarkers to know the extent of disease severity so that timely action can be taken to reduce mortality. We aimed to determine the usefulness of C-reactive protein (CRP) levels in assessing COVID-19 disease severity and correlate them with mortality due to the same. Methods: Data for COVID-19 were retrospectively collected and analyzed from May 2020 to October 2020. The CRP value was correlated with disease severity using Karl Pearson's correlation coefficient. A logistic regression model was adopted to analyze the association between mortality and related factors. Results: Out of 642 patients enrolled, 22 died while 620 recovered. Most of the non-survivors were male. Multivariate analysis showed that age, diabetes, hypertension, and CRP values were significantly associated with mortality. CRP showed a strong positive correlation with disease severity and, hence, mortality. Conclusion: In patients with COVID-19, CRP correlated with disease severity and tended to be a good predictor of adverse outcome.

Key words: Coronavirus disease-2019, C-reactive protein, Prognostic marker, Severe acute respiratory syndrome coronavirus 2, Cytokines

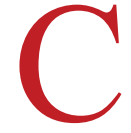

oronavirus belongs to the family Coronaviridae, subfamily Orthocoronavirinae, and order Nidovirales. This novel virus was named as severe acute respiratory syndrome coronavirus 2 (SARS-CoV-2) by the WHO because of its similarity to SARS-CoV in its gene sequence. The first case of pneumonia caused by this virus was reported in Wuhan, China, in December 2019 [1]. The situation eventually took the form of a global pandemic. For the patients infected by SARS-CoV-2, it has been seen that some do not show signs of respiratory distress during the course of the disease, indicating the multifaceted nature of coronavirus disease-2019 (COVID-19) [1]. It has been observed that a number of patients with mild symptoms have suddenly progressed to severe illness [2]. Hence, it is important to timely diagnose and treat all patients to reduce the fatality rate and, hence, to improve the outcome. For this, a reliable and convenient diagnostic serological marker is essential to predict the severity of disease. A number of clinical studies have shown that altered levels of certain serological markers may be associated with disease severity [3-5]. Out of these markers, C-reactive protein (CRP) has been determined to be a valuable parameter which alters to significant levels in severe cases of COVID-19 disease [5]. CRP is an important acutephase reactant that can rise up to significant levels soon after the onset of inflammation, tissue injury, or cell damage. The raised CRP levels may be associated with the production of cytokines in complicated cases of disease caused by this virus. Just like SARS, patients critical of COVID-19 presented with raised plasma cytokine levels, suggesting the involvement of an inflammatory storm in disease pathogenesis [6]. CRP, a routinely measured inflammatory marker, was increased in most patients with COVID-19 and was also associated with disease severity [6-8]. Trends and threshold levels of CRP in severe cases of COVID-19 illness, if defined from a prognostic point of view, may help in risk stratification, guidance of clinical management of COVID-19 patients, and also help in monitoring of resource utilization. In this retrospective study, we aimed to determine the potential of CRP in outcome prediction of patients with COVID-19.

\section{METHODS}

Study Design and Participants

This retrospective, single-center study was conducted at Sharda Hospital, Greater Noida, from May 2020 to October 2020. Sharda Hospital was requisitioned as a designated hospital for COVID-19. All patients with COVID-19 disease who had a clinical outcome (died or recovered) through May 2020 were enrolled in the study. Patients without CRP detection were excluded from the study. The confirmation 
of severity of patients with COVID-19 was made according to guidelines issued by the Ministry of Health and Family Welfare, Directorate General of Health Services, Government of India. The study was approved by the Institutional Ethics Committee. Written consent was not required because of the retrospective nature of the study.

\section{Data Collection}

Information for each patient was obtained mainly by screening the health records and laboratory information systems provided by the hospital software. Epidemiological information including age, gender, underlying chronic diseases, and history of smoking and drinking was reviewed and assessed. The category of severity of COVID-19 and its corresponding CRP values was also reviewed and recorded.

\section{Statistical Analysis}

Statistical data analysis was performed using SPSS-21 software. Categorical variables were presented as numbers (\%). The Chisquare test was utilized to compare differences in categorical variables. Karl Pearson's coefficient of correlation was used to analyze the correlation. $\mathrm{p}<0.05$ was considered statistically significant. Multivariate analysis was carried out using a logistic regression model to analyze the association between mortality and related factors. Demographic factors (age and gender) and other variables, including comorbidities, were analyzed in multivariate analysis.

\section{RESULTS}

A total of 642 patients were enrolled in the study, which included 515 cases of mild illness, 78 cases of moderate illness, and 49 of severe illness. Out of the total patients, 620 recovered while 22 patients died of COVID-19. There were 398 males and 244 females, with an age ranging from 10 to 85 years. There were 126 cases of hypertension, 104 cases of diabetes, 64 cases of coronary disease, 38 cases of chronic pulmonary disease, and 48 cases of chronic renal disease. Although most patients who died were male, no significant difference was observed between gender and mortality $(\mathrm{p}>0.05)$. Age also had no significant effect on mortality $(p>0.05)$. One hundred and eighteen $(18.4 \%)$ patients had a history of smoking. No significant difference existed in the mortality rate of smokers and nonsmokers $(p>0.05)$. Compared to survivors, the non-survivors had a significantly higher incidence of chronic diseases such as hypertension $(\mathrm{p}<0.05)$, diabetes $(\mathrm{p}<0.05)$, chronic pulmonary disease $(p<0.05)$, and coronary disease $(p<0.05)$, as shown in Table 1. However, no significant difference was observed between the survivors and non-survivors with chronic renal disease ( $p>0.05)$.

As indicated in Table 2, a strong positive correlation ( $\mathrm{r}=0.989)$ was found between the values of CRP and mortality, which indicates that as the value of CRP increased, the chances of nonsurvival of patients also increased. Hence, it can be deduced that CRP is a good prognostic marker for COVID-19 disease progression.

On multivariate analysis, it was observed that age $(95 \%$ CI, 1.92-26.228; $\mathrm{p}=0.003$ ), diabetes (95\% CI, 0.01-0.372; $\mathrm{p}=0.002$ ), hypertension (95\% CI, 1.347-10.927; $\mathrm{p}=0.011$ ), and CRP (95\% CI, 0.094-0.899; $\mathrm{p}=0.032)$ were significantly associated with mortality of COVID-19 patients, as shown in Table 3.

On the basis of the above-mentioned facts, it can be deduced that CRP is a good prognostic marker in COVID-19 disease progression and, hence, can be used to predict the outcome of the disease.

Table 1: Epidemiological findings of patients with COVID-19

\begin{tabular}{|c|c|c|c|c|}
\hline Finding & Total patients $(n=642)$ & Survivors $(n=620)$ & Non-survivors $(n=22)$ & p value \\
\hline Age (years) & & & & 0.101 \\
\hline$<45$ & 398 & 391 & 7 & \\
\hline $45-60$ & 154 & 146 & 8 & \\
\hline$>60$ & 90 & 83 & 7 & \\
\hline Gender & & & & 0.291 \\
\hline Males & $398(62.0 \%)$ & $382(61.6 \%)$ & $16(72.7 \%)$ & \\
\hline Females & $244(38.0 \%)$ & $238(38.4 \%)$ & $6(27.3 \%)$ & \\
\hline Smoking & $118(18.4 \%)$ & $114(18.4 \%)$ & $4(18.2 \%)$ & 0.799 \\
\hline \multicolumn{5}{|l|}{ Comorbidities } \\
\hline Hypertension & $126(19.6 \%)$ & $116(18.7 \%)$ & $11(50.0 \%)$ & $0.000 *$ \\
\hline Diabetes & $104(16.2 \%)$ & $100(16.1 \%)$ & $4(18.2 \%)$ & $0.041 *$ \\
\hline Coronary disease & $64(10.0 \%)$ & $58(9.4 \%)$ & $6(27.3 \%)$ & $0.006^{*}$ \\
\hline Chronic pulmonary disease & $38(6.0 \%)$ & $34(5.5 \%)$ & $4(18.2 \%)$ & $0.043^{*}$ \\
\hline Chronic renal disease & $48(7.5 \%)$ & $42(6.8 \%)$ & $7(31.8 \%)$ & 0.063 \\
\hline
\end{tabular}


Table 2: Values of $\mathrm{C}$-reactive protein in relation to survival of COVID-19 patients

\begin{tabular}{lcccc}
\hline $\begin{array}{l}\text { CRP value } \\
(\mathbf{m g} / \mathbf{L})\end{array}$ & $\begin{array}{c}\text { Total } \\
\text { patients } \\
(\mathbf{n}=\mathbf{6 4 2})\end{array}$ & $\begin{array}{c}\text { Survivors } \\
(\mathbf{n}=\mathbf{6 2 0})\end{array}$ & $\begin{array}{c}\text { Non- } \\
\text { survivors } \\
(\mathbf{n}=\mathbf{2 2})\end{array}$ & $\begin{array}{c}\text { Karl Pearson's } \\
\text { correlation } \\
\text { coefficient (r) }\end{array}$ \\
\hline$\leq 24$ & 482 & 482 & 0 & 0.989 \\
$>24-48$ & 77 & 70 & 7 & \\
$>48-96$ & 83 & 68 & 15 & \\
\hline
\end{tabular}

Table 3: Multivariate analysis of factors related to mortality in COVID-19 patients

\begin{tabular}{lcc}
\hline Variables & $\mathbf{9 5 \%}$ confidence interval & p value \\
\hline Age & $1.92-26.228$ & $0.003 *$ \\
Gender & $0.157-1.311$ & 0.144 \\
Diabetes & $0.01-0.372$ & $0.002 *$ \\
Hypertension & $1.347-10.927$ & $0.011^{*}$ \\
Coronary disease & $0.363-6.164$ & 0.575 \\
Chronic pulmonary & $0.388-9.903$ & 0.415 \\
disease & & \\
Chronic renal disease & $1.367-19.349$ & 0.065 \\
C-reactive protein & $0.094-0.899$ & $0.032 *$ \\
\hline
\end{tabular}

Multivariate analysis was carried out using a logistic regression model. *Statistically significant

\section{DISCUSSION}

In this retrospective study, we tried to evaluate the significance of CRP in assessing the severity of COVID-19 illness. We also assessed the severity of disease as mild, moderate, and severe as per guidelines and correlated it with different epidemiological parameters and comorbidities.

On analyzing the possible factors associated with disease severity and mortality, it was found that comorbidities such as hypertension and diabetes along with age of patients and their CRP values were significantly associated with disease severity and hence mortality. This was in agreement with a study done in China where age and CRP were significantly associated with disease severity [9]. However, diabetes and hypertension were not significant contributing factors to disease severity according to that study [9]. On the other hand, a meta-analysis done in Brazil showed a positive association between diabetes and hypertension and COVID-19 severity and mortality [10].

In our study, we observed that the COVID-19 patients showed elevated CRP levels, which is in agreement with various other studies $[5,11,12]$. According to our study, CRP levels can well be associated with disease severity. This is in accordance with a study which reported that patients with severe disease had an average CRP concentration of $39.4 \mathrm{mg} / \mathrm{L}$ whereas those with mild symptoms had a CRP concentration of $18.8 \mathrm{mg} / \mathrm{L}$ [13]. Another study also reported increased levels of CRP in the severe group at the initial stage than those in the mild group [3]. The same study also reported a significant association between the concentration of CRP and the aggravation of non-severe patients with COVID-19 [3]. In another study also, it was found that the mean concentration of CRP was significantly higher in severe patients $(46 \mathrm{mg} / \mathrm{L})$ than in non-severe patients $(23 \mathrm{mg} / \mathrm{L})$ [14]. Another study conducted in China showed that the patients who died due to COVID-19 had about 10-fold higher levels of CRP than the survivors (median 100 vs. $9.6 \mathrm{mg} / \mathrm{L}$ ) [9].

This study has few limitations. First, the relatively small number of patients limits the conclusions. For example, several indicators can be related to disease severity in univariate regression analysis, but only a few indicators, including CRP, remain significant in multivariate analysis. Future research should focus on these indicators as well. Second, the progression of disease is generally a dynamic process; thus, the CRP values which we have collected only at the time of admission to the hospital may not accurately reflect change in the condition. For that, time-to-time analysis of biomarkers is required. Furthermore, there is a need to evaluate the association between COVID-19 severity and other serological markers such as procalcitonin, lactate dehydrogenase, and ferritin which we have not done in this study.

\section{CONCLUSION}

It can be concluded from the above discussion that CRP levels are correlated well with the severity of COVID-19 illness. Therefore, it may be a suitable marker for assessing a patient's condition along with other clinical parameters. Elevated CRP levels in COVID-19 patients might be an indication of high inflammatory stress and, hence, contribute toward severe illness or even death. Therefore, patients with significantly elevated CRP levels should be provided prompt attention and adequate treatment. However, the exact role of CRP in patients with COVID-19 still remains to be determined.

\section{REFERENCES}

1. Chen W, Zheng KI, Liu S, et al. Plasma CRP level is positively associated with the severity of COVID-19. Ann Clin Microbiol Antimicrob 2020;19:18.

2. Chen N, Zhou M, Dong X, et al. Epidemiological and clinical characteristics of 99 cases of 2019 novel coronavirus pneumonia in Wuhan, China: A descriptive study. Lancet 2020;395:507-13.

3. Tan C, Huang Y, Shi F, et al. C-reactive protein correlates with computed tomographic findings and predicts severe COVID-19 early. J Med Virol 2020;92:856-62.

4. Tian W, Jiang W, Yao J, et al. Predictors of mortality in hospitalized COVID-19 patients: A systematic review and meta-analysis. J Med Virol 2020;92:1875-83.

5. Wang $\mathrm{G}, \mathrm{Wu} \mathrm{C}$, Zhang Q, et al. C reactive protein level may predict the risk of COVID-19 aggravation. Open Forum Infect Dis 2020;7:ofaa153.

6. Qin C, Zhou L, Hu Z, et al. Dysregulation of immune response in patients with COVID-19 in Wuhan, China. Clin Infect Dis 2020;71:762-8.

7. Zhang JJ, Dong X, Cao YY. Clinical characteristics of 140 patients infected by SARS-CoV-2 in Wuhan, China. Allergy. 2020;75:1730-41.

8. Yang W, Cao Q, Qin L, et al. Clinical characteristics and imaging manifestations of the 2019 novel coronavirus disease (COVID-19): A multicenter study in Wenzhou City, Zhejiang, China. J Infect 2020;80:388-93.

9. Luo X, Zhou W, Yan X, et al. Prognostic value of C-reactive protein in patients with coronavirus 2019. Clin Infect Dis 2020;71:2174-9.

10. de Almeida-Pititto B, Dualib PM, Zajdenverg L, et al. Severity and mortality of COVID 19 in patients with diabetes, hypertension and cardiovascular disease: A meta-analysis. Diabetol Metab Syndr 2020;12:75. 
11. Zhu N, Zhang D, Wang W, et al. A novel coronavirus from patients with pneumonia in China, 2019. N Engl J Med 2020;382:727-33.

12. Wang L. C-reactive protein levels in the early stage of COVID-19. Méd Mal Infect 2020;50:332-4.

13. Gao Y, Li T, Han M, et al. Diagnostic utility of clinical laboratory data determinations for patients with the severe COVID-19. J Med Virol 2020;92:791-6.

14. Mo P, Xing Y, Xiao Y, et al. Clinical characteristics of refractory COVID-19 pneumonia in Wuhan, China. Clin Infect Dis. 2020;2020:ciaa270.
Funding: None; Conflicts of Interest: None Stated.

How to cite this article: Sarwat T, Kumar D, Singh NP, Kakru D. C-reactive protein as a prognostic marker in coronavirus disease-2019. Eastern J Med Sci. 2021;6(3):46-49.

DOI: 10.32677/EJMS.2021.v06.i03.3034 